\title{
La salud oral en la dentición primaria: (II) Estudio sobre la maloclusión y otras anomalías orales en una muestra de 441 niños de Vigo
}

\author{
Iglesias Parada IM*
}

\section{RESUMEN}

La intención de este estudio, que se dividió en tres partes, es valorar la salud oral en los niños de Vigo con dentición primaria. En esta segunda parte se estudian la prevalencia de la maloclusión y otras anomalías orales. MUESTRA: 441 niños entre los 14 y 92 meses de edad (edad media 59,6 meses), todos ellos con dentición primaria al inicio del estudio. RESULTADOS: (precisión de $\pm 4,6 \%$ para una seguridad del $95 \%$ ) el $90,92 \%$ de la muestra es ortognática, el $8,17 \%$ es retrognática y el 0,91 es prognática; la prevalencia de la maloclusión es de 39,2\%, siendo leve en el 21,3\% moderada en el 14,5\% y servera en el 3,4\% de la muestra; las maloclusiones más frecuentes son los apiñamientos, las mordidas abiertas anteriores y las mordidas cruzadas. Las diversas anomalías oralesa afectan a un $6,57 \%$ de la muestra. CONCLUSIÓN: El diagnóstico precoz de la maloclusión admite la posibilidad de planificar el tratamiento según las necesidades del niño. Las anomalías dentales en la dentición temporal advierten sobre la contingencia de encontrar la misma anomalía o más severa en la dentición permanente.

Palabras clave: Epidemiología. Dentición primaria. Maloclusión. Anomalía oral. Prevalencia.

\section{ABSTRACT}

The purpose of this three-part research is to evaluate the oral health of children from Vigo with temporary dentition. Prevalences of Malocclusion and other oral anomalies. Sample: 441 children, aged between fourteen and ninety-two months (average age: 59,6 months), all of them with milk teeth at the beginning of the research. Results: (4,6\% of precesion for a $95 \%$ of confidence), the 90,92 per cent of the sample is orthognatic, the 8,17 per cent is retrognatic and the 0,91 is prognatic. The prevalence of malocclusion is 39,2 percent. It is 21,3 per cent when slight, 14,5 per cent when mild and the 3,4 per cent of the sample is severe. The most frecuent malocclusions are crowded together theet, open front bite and crossed bites. Diverse oral anomalies affect the 6,57 per cent of the sample. Conclusion: A quick diagnosis of malocclusion lets the possibility of planning a treatment depending on the child needs. Dental anomalies in temporary dentition warn about finding the same or worse problem in permanent dentition.

Key words: Epidemiology, Primary Dentition, Malocclusion, Oral Anomaly, Prevalence.

* Estomatólogo Interino del SERGAS. Centro de Atención Primaria de Coia. Unidad de Salud Bucodental. Vigo.

Iglesias Parada IM. La salud oral en la dentición primaria: (II) Estudio sobre la maloclusión y otras anomalías orales en una muestra de 441 niños de Vigo. Av. Odontoestomatol 2003; 19-1: 11-19 


\section{INTRODUCCIÓN}

En una encuesta nacional realizada en 1997, la percepción de los padres sobre los problemas oclusales de los niños fue muy baja: rondó en torno al $7 \%$. Alrededor del $65 \%$ de los niños nunca visitaron un dentista antes de los 6 años. La opinión de los expertos, aunque no de forma homogénea, es que los problemas de malposición dentaria se mantendrán; si bien, también, se prevé una mejor asistencia odontológica en la población infantil (1).

Aunque actualmente los resultados sobre la prevalencia y las necesidades de tratamiento comienzan a homogeneizarse, para Riobóo una de las carencias que se da es la falta de unanimidad de criterios en los diferentes índices empleados para evaluar las malposiciones dentarias y sus necesidades de tratamiento (2).

La intención de este estudio es aportar datos epidemiológicos sobre la prevalencia de la maloclusión y otras anomalías orales congénitas en la infancia temprana.

\section{METODOLOGÍA}

\section{Características técnicas:}

- Universo: Población de niños con dentición primaria de Vigo.

- Muestra: El estudio se emplearon 441 niños, de 14 a 92 meses de edad, que acudieron a la Unidad de Salud Bucodental-1 del Centro de Atención Primaria de Coia en Vigo, desde marzo de 1998 hasta diciembre de 2001. Se exigió como único requisito exhibir dentición primaria al abrir la historia clínica.

- Selección de la muestra: Muestreo no probabilístico (3), integran el estudio la totalidad de niños con dentición primaria historiados.

- Error de muestreo: La precisión fue de $\pm 4,6 \%$ para una seguridad del $95 \%$ en las maloclusiones ( $p=$ $0,5)$. En las otras anomalías orales, debido a una prevalencia muy baja, el error de muestreo disminuyó manteniendo la misma seguridad (4).
- Recogida de datos: Se realiza una entrevista estructurada a los acompañantes para recabar los datos de interés. A los niños se les explora en un sillón dental con luz artificial.

- Estudio: Retrospectivo transversal.

\section{Definición de las variables:}

En la primera parte de este estudio ya se expusieron los criterios empleados para definir las variables: caries dental, higiene oral y pautas de alimentación de la primera infancia. En esta ocasión se definen las siguientes variables.

- Oclusión: en sentido mesiodistal, los dientes superiores ocluyen con el antagonista inferior y el distal a éste. Las excepciones son los incisivos centrales inferiores que únicamente ocluyen con los centrales superiores y los segundos molares superiores que ocluyen con los segundos inferiores terminando en un mismo plano. En esta dentición la sobremordida es mayor, pudiendo cubrir casi completamente los incisivos superiores a los inferiores. En sentido vestibulolingual las piezas superiores sobrepasan por vestibular a las inferiores (5).

- Criterios de severidad de la maloclusión: se valora la relación de los dientes el plano anteroposterior y en el plano transversal. En función de estas relaciones se aplican unos grados de severidad:

Leve: En el plano anteroposterior el resalte es menor de $5 \mathrm{~mm}$, la mordida abierta menor de $3 \mathrm{~mm}$ o la mordida es borde a borde. En plano transversal la falta de espacio es menor de 1/3 del ancho mesiodistal mayor del diente. Si existe mordida cruzada, debe de ser unilateral y no afectarse la guía canina.

Moderado: En el plano anteroposterior el resalte es mayor de 5 y menor de $10 \mathrm{~mm}$, si hay mordida abierta es mayor de 3 y menor de $10 \mathrm{~mm}$ o el resalte es negativo sin discrepancia ósea. En plano transversal la falta de espacio es mayor de $1 / 3$ y menor de 2/3 del ancho mesiodistal mayor del diente. Si existe mordida cruzada, debe de ser unilateral y afectar a la guía canina, o bien bilateral sin afectar las guías caninas.

Severo: En el plano anteroposterior el resalta es 
TABLA 1.- ESTUDIO SOBRE LA PREVALENCIA DE LA MALOCLUSIÓN Y SUS DISTINTOS TIPOS EN LA MUESTRA Y POR SEXOS. Muestra: 441 niños. Vigo 1998-2001

\begin{tabular}{|c|c|c|c|}
\hline PREVALENCIAS POR SEXO Y TOTAL & $\begin{array}{c}\text { NIÑO/NIÑA } \\
247 / 194\end{array}$ & $\begin{array}{l}\text { PORCENTAJES } \\
\text { PARCIALES } \\
\text { NIÑO/NIÑA } \\
\end{array}$ & $\begin{array}{c}\text { PORCENTAJE } \\
\text { TOTAL } \\
\mathrm{N}=441 \\
\end{array}$ \\
\hline $\begin{array}{l}\text { NORMOCLUSIÓN } \\
\text { MALOCLUSIÓN }\end{array}$ & $\begin{array}{c}154 / 114 \\
93 / 80\end{array}$ & $\begin{array}{l}62,3 \% / 58,8 \% \\
37,7 \% / 41,2 \% \\
\end{array}$ & $\begin{array}{l}60,8 \% \\
39,2 \% \\
\end{array}$ \\
\hline $\begin{array}{l}\text { MALOCLUSIÓN CLASE I } \\
\text { 1- Alteraciones sagitales } \\
\text { - Apiñamiento } \\
\text { - Mordida cruzada anterior } \\
\text { - Mordida abierta anterior } \\
\text { - Mordida borde a borde } \\
\text { - Resalte } \\
\text { - Sobremordida } \\
\text { 2- Alteraciones transversales } \\
\text { - Cruzada posterior bilateral } \\
\text { - Cruzada posterior derecha } \\
\text { - Cruzada posterior izquierda } \\
\text { 3- Alteraciones combinadas } \\
\text { - Apiñamiento/mordida cruzada } \\
\text { - derecha } \\
\text { - izquierda } \\
\text { - Mordida abierta/mordida cruzada } \\
\text { - bilateral } \\
\text { - izquierda } \\
\text { CLASE 2/1 } \\
\text { 1- Alteraciones sagitales } \\
\text { - Sin apiñamiento } \\
\text { - Con apiñamiento } \\
\text { - Mordida abierta anterior } \\
\text { 2- Alteraciones transversales } \\
\text { - Derecha } \\
\text { 3- Alteraciones combinadas } \\
\text { - Apiñamiento/mordida cruzada } \\
\text { - bilateral } \\
\text { - Sin apiñamiento }\end{array}$ & $\begin{array}{c}30 / 26 \\
2 / 0 \\
17 / 10 \\
1 / 1 \\
2 / 2 \\
1 / 1 \\
\\
1 / 1 \\
7 / 7 \\
8 / 7\end{array}$ & $\begin{array}{c}12,1 \% / 13,4 \% \\
0,8 \% / 0,0 \% \\
6,9 \% / 5,2 \% \\
0,4 \% / 0,5 \% \\
0,8 \% / 1,0 \% \\
0,4 \% / 0,5 \% \\
0,4 \% / 0,5 \% \\
2,8 \% / 3,6 \% \\
3,2 \% / 3,6 \% \\
\\
0,8 \% / 0,0 \% \\
0,0 \% / 0,5 \% \\
1,2 \% / 0,5 \% \\
0,4 \% / 0,5 \% \\
\\
5,3 \% / 7,7 \% \\
0,8 \% / 0,0 \% \\
0,4 \% / 1,0 \% \\
0,4 \% / 0,5 \%\end{array}$ & $\begin{array}{r}12,6 \% \\
0,5 \% \\
6,1 \% \\
0,5 \% \\
0,9 \% \\
0,5 \% \\
\\
0,5 \% \\
3,2 \% \\
3,4 \%\end{array}$ \\
\hline
\end{tabular}

mayor de $10 \mathrm{~mm}$, la mordida abierta también es mayor de $10 \mathrm{~mm}$ o el resalte es negativo por discrepancia ósea. En plano transversal la falta de espacio es mayor de 2/3 del ancho mesiodistal mayor del diente. Si existe mordida cruzada, debe de ser bilateral y afectar ambas guías caninas.
- Síndrome de obstrucción respiratoria, entendida por aquella disposición que favorezca la respiración oral, teniéndose en consideración las siguientes causas (6):

- Patología quirúrgica cuando se realizó una amigdalectomía, una adenoidectomía, una miringotomía 


\section{TABLA 2.- ESTUDIO SOBRE LA PREVALENCIA DE LA MALOCLUSIÓN Y SU SEVERIDAD EN LA} MUESTRA Y POR SEXOS. Muestra: 441 niños. Vigo 1998-2001

\begin{tabular}{|l|c|c|c|c|}
\hline & MUESTRA & NIÑOS & NIÑAS & P (1)=0,2798 \\
\hline Oclusión normal & $268(60,8 \%)$ & $154(62,3 \%)$ & $114(58,8 \%)$ & \\
Maloclusión leve & $94(21,3 \%)$ & $56(22,7 \%)$ & $38(19,6 \%)$ & \\
Maloclusión moderada & $64(14,5 \%)$ & $28(11,3 \%)$ & $36(18,6 \%)$ & $P(2)=0,0659$ \\
Maloclusión severa & $15(3,4 \%)$ & $9(3,6 \%)$ & $6(3,1 \%)$ & \\
\hline TOTAL & $441(\mathbf{1 0 0 \%})$ & $\mathbf{2 4 7 ( 1 0 0 \% )}$ & $\mathbf{1 9 4 ( 1 0 0 \% )}$ & \\
\hline
\end{tabular}

1. Prueba de Mann-Whitney: no hay dismorfismo sexual en la prevalencia de maloclusiones.

2. Prueba de Mantel-Haenszel: parece que hay una tendencia a diferenciarse por sexos las maloclusiones moderadas,

\begin{tabular}{|l|c|c|c|c|}
\hline \multicolumn{5}{|c|}{ TABLA 3.- ESTUDIO SOBRE LA PREVALENCIA DE LA MALOCLUSIÓN EN LA MUESTRA Y POR } \\
SEXOS DISTRIBUIDA POR EDADES. Muestra: 441 niños. Vigo 1998-2001
\end{tabular}

1. Prueba de Mann-Whitney: no hay dismorfismo sexual.

o cualquier combinación entre ellas.

- Patología médica que favorezca la respiración bucal como la rinitis alérgica y/o el asma. Se descarta como patología médica aquella que termina posteriormente siendo causa de intervención quirúrgica.

- Patología médico-quirúrgica cuando se presentan ambas circunstancias.

- Anomalías orales: son aquellas no admitidas que se observan en la exploración intraoral.

Los resultados son procesados en el programa SPSS 6.0 para Windows. Se manifiesta significación estadística cuando los valores de las pruebas de MannWhitney, de Mantel-Haenszel y el análisis de la varianza Kruskal-Wallis son $\leq 0,05$.

\section{RESULTADOS}

La muestra la componen 441 niños, con una edad media de 59,66 meses, 247 son niños (56\%) y 194 son niñas (44\%). Se dieron diferencias a favor de los niños en la proporción que compone la muestra $(\mathrm{p}=$ $0,0116)$. Esta diferencia fue a causa del grupo de los seis años de edad ( $p=0,0071)$.

En este estudio el $90,92 \%$ de los niños fueron ortognáticos, el $8,15 \%$ fueron retrognáticos y el $0,91 \%$ fueron prognáticos. El 39,2\% de los niños presentaron anomalías oclusales en función del patrón de oclusión definido. En la Tabla 1 se exponen los distintos tipos de maloclusiones en la muestra y por sexos. Las más frecuentes en orden decreciente fueron: los apiñamientos dentales, las mordidas abiertas, las clases 2-1 de Angle y las mordidas cruzadas unilaterales, el resto tiene una frecuencia similar muy baja. Cuando se estudió el comportamiento de las distintas maloclusiones relacionándolas con el sexo no se observaron diferencias $(p=0,5280)$.

A las maloclusiones, también se las clasificó con relación a unos criterios de severidad. La distribución de 


\begin{tabular}{|c|c|c|c|}
\hline \multicolumn{4}{|c|}{$\begin{array}{l}\text { TABLA 4.- ESTUDIO DE LA RELACIÓN DE LA } \\
\text { MALOCLUSIÓN EN LA MUESTRA Y POR SEXOS CON LA } \\
\text { ALIMENTACIÓN INFANTIL, LA HIGIENE DENTAL, LA } \\
\text { CARIES DENTAL Y EL SÍNDROME DE OBSTRUCCIÓN } \\
\text { RESPIRATORIA; Y ASIMISMO CON LA SEVERIDAD DE LA } \\
\text { MALOCLUSIÓN. } \\
\text { Muestra: } 441 \text { niños. Vigo } 1998-2001\end{array}$} \\
\hline & $\begin{array}{l}\text { MUE } \\
\mathrm{N}=\end{array}$ & $\begin{array}{l}\text { NIÑOS } \\
\mathrm{N}=121\end{array}$ & $\begin{array}{l}\text { NIÑAS } \\
\mathrm{N}=92\end{array}$ \\
\hline $\begin{array}{l}\text { Alimentación infancia } \\
\text { Higiene dental } \\
\text { Caries dental P } \text { (1) }^{(1)} \\
\text { Síndrome obstrucción } \\
\text { respiratoria }\end{array}$ & $\begin{array}{l}0,0225 \\
0,3088 \\
0,4360 \\
0,8166\end{array}$ & $\begin{array}{c}\text { Severidad } \\
\text { maloclusión } \\
P^{(1)} \\
0,3071 \\
0,0634 \\
0,1653 \\
0,7928\end{array}$ & $\begin{array}{l}0,0861 \\
0,8212 \\
0,2849 \\
0,6858\end{array}$ \\
\hline $\begin{array}{l}\text { Alimentación infancia } \\
\text { Higiene dental } \\
\text { Caries dental P }{ }^{(1)} \\
\text { Síndrome obstrucción } \\
\text { respiratoria }\end{array}$ & $\begin{array}{l}0,0169 \\
0,0666 \\
0,1828 \\
0,5320\end{array}$ & $\begin{array}{c}0,2811 \\
0,0211 \\
0,1904 \\
0,3682 \\
P^{(1)} \\
\text { maloclusión }\end{array}$ & $\begin{array}{l}0,0211 \\
0,8638 \\
0,6139 \\
0,9782\end{array}$ \\
\hline
\end{tabular}

1. Análisis de la varianza de Kruskal-Wallis.

las mismas fue la siguiente: el $54,33 \%$ de ellas fueron leves, el 36,99\% moderadas y el 8,67\% severas. En la tabla 2 se pueden comparar las prevalencias relativas en la muestra y por sexos. Globalmente no se apreciaron diferencias en las severidades de las anomalías con relación al sexo $(p=0,2798)$, aunque parece que existe una tendencia a presentar más anomalías moderadas las niñas que los niños $(p=0,065)$.

En la muestra, al comparar la prevalencia por años, la tendencia es a disminuir conforme aumenta la edad a partir de los 2 años. Un comportamiento aproximado se observa en el grupo de los niños, sin embargo, el patrón en las niñas no está tan definido. A pesar de esto, no se manifestaron diferencias con relación al sexo ni en la prevalencia de las maloclusiones en su conjunto ( $p=0,4445)$, ni por grupos de edad distintos (Tabla 3).

En un estudio anterior sobre la dentición mixta con
1.032 niños de 8,3 años de edad media, se relacionó a la maloclusión con las caries de los molares temporales, con la alimentación en biberón y con las niñas. No se encontró relación con el síndrome de obstrucción respiratoria (6). Ahora se pretendió realizar un estudio similar con la dentición primaria. Se encontró en la muestra una relación entre la maloclusión y el tipo de alimentación en la primera infancia ( $p=0,0169$ ), los niños alimentados con biberón manifestaron significativamente más maloclusiones. Al segregarlo por sexos, sólo se alcanzó significación en las niñas $(p=0,0211)$. En la muestra se anunció una relación entre las maloclusiones y la higiene dental $(p=0,0666)$ que sólo se pudo confirmar en los niños ( $p-0,0211)$, pero no en las niñas. La higiene oral fue peor en los casos de maloclusiones dentales. En esta dentición no se observó ninguna asociación entre la maloclusión y la caries dental, ni tampoco con el síndrome de obstrucción respiratoria (Tabla 4).

En este mismo proyecto se realizó un estudio sobre la prevalencia de otras anomalías orales. Se encontró que el $6,57 \%$ de la muestra presentó alguna anomalía. En la Tabla 5 se enumeran dichas anomalías, sus frecuenciasd y sus prevalencias. No se acusaron diferencias con relación al sexo en estas anomalías ( $p=0,2758$ ), aunque fueron algo más habituales en los niños. Las anomalías dentales más frecuentes fueron: los dientes supernumerarios y las agenesias dentales, con una prevalencia de $1,13 \%$ y $0,90 \%$ respectivamente. La anomalía oral más común fue el frenillo en el labio superior con una prevalencia del $1,13 \%$.

\section{DISCUSIÓN}

En la revisión de la bibliografía para conocer la prevalencia de la maloclusión en la dentición primaria se confirmó la ausencia de unanimidad y homogeneización de criterios, algo que ya expresó Riobóo (2). Se encontraron estudios con una prevalencia desde el $78 \%$ (7) hasta otros con el $28,5 \%$ (8). Este estudio pre- 


\begin{tabular}{|c|c|c|c|c|c|c|}
\hline \multicolumn{7}{|c|}{$\begin{array}{l}\text { TABLA 5.- FRECUENCIAS DE LAS ANOMALÍAS ORODENTALES. } \\
\text { Muestra: } 441 \text { niños. Vigo 1998-2001 }\end{array}$} \\
\hline & $\begin{array}{c}\text { MUESTRA } \\
\mathrm{N}=441\end{array}$ & $\begin{array}{l}\text { PREVA- } \\
\text { LENCIA }\end{array}$ & $\begin{array}{l}\text { NIÑOS } \\
\mathrm{N}=247\end{array}$ & $\begin{array}{l}\text { PREVA- } \\
\text { LENCIA }\end{array}$ & $\begin{array}{l}\text { NIÑAS } \\
\mathrm{N}=194\end{array}$ & $\begin{array}{l}\text { PREVA- } \\
\text { LENCIA }\end{array}$ \\
\hline Agenesia dental: & & $0,90 \%$ & & $0,80 \%$ & & $1,03 \%$ \\
\hline Lateral superior & 2 & & 1 & & 1 & \\
\hline Lateral inferior & 2 & & 1 & & 1 & \\
\hline Supernumerario: & & $1,13 \%$ & & $1,61 \%$ & & $0,51 \%$ \\
\hline Central superior & 1 & & 1 & & 0 & \\
\hline Laterales superiores & 2 & & 2 & & 0 & \\
\hline Lateral superior & 1 & & 0 & & 1 & \\
\hline Lateral inferior & 1 & & 1 & & 0 & \\
\hline Hipolasia esmalte: & & & & & & \\
\hline Centrales superiores & 2 & $0,45 \%$ & 2 & $0,80 \%$ & 0 & $0,00 \%$ \\
\hline Fusión dental: & & & & & & \\
\hline Canino y lateral inferior & 1 & $0,22 \%$ & 0 & $0,00 \%$ & 1 & $0,51 \%$ \\
\hline Hipodoncia dental: & & & & ค & 0 & $000 \%$ \\
\hline Lateral superior & 1 & $0,22 \%$ & 1 & $\begin{array}{l}0,40 \% \\
0,40 \%\end{array}$ & 0 & $0,00 \%$ \\
\hline $\begin{array}{l}\text { Diente natal } \\
\text { ANOMALIAS ORALES }\end{array}$ & 1 & $\frac{0,22 \%}{3,40 \%}$ & 1 & $3,40 \%$ & & $3,09 \%$ \\
\hline Frenillo labio superior & 5 & $1,13 \%$ & 3 & $1,21 \%$ & 2 & $1,03 \%$ \\
\hline Lengua geográfica & 4 & $0,90 \%$ & 2 & $0,80 \%$ & 2 & $1,03 \%$ \\
\hline Paladar ojival & 3 & $0,68 \%$ & 1 & $0,40 \%$ & 2 & $1,03 \%$ \\
\hline Frenillo lingual & 2 & $0,45 \%$ & 2 & $0,80 \%$ & 0 & $0,00 \%$ \\
\hline Melanosis oral & 1 & $0,22 \%$ & 1 & $0,40 \%$ & 0 & $0,00 \%$ \\
\hline TOTAL & 29 & $6,57 \%$ & 19 & $7,69 \%$ & 10 & $5,15 \%$ \\
\hline
\end{tabular}

sentó una prevalencia del $39,2 \%$ de niños con maloclusiones. Se dan estudios con prevalencias algo superiores, como en el caso de escolares cordobeses de $1^{\circ}$ de EGB con un 46,26\% (9). O bien, estudios de otros países, como en Istria (Croacia), donde el $46,95 \%$ de los niños con dentición primaria presentaron alteraciones ortodónticas (10).

En la Tabla 3 se puede ver que el patrón en la prevalencia de las maloclusiones tendió a disminuir a partir de los 3 años de edad. Esto fue muy acusado en los niños. Cabría preguntarse si en esto influye el cambio en las dimensiones del arco dentario que se produce durante el crecimiento del infante en estas edades (11).

También se encuentran discrepancias entre las distintas maloclusiones. En la Tabla 6 se exponen unos estudios sobre sus frecuencias en niños con dentición primaria. Sin embargo, a excepción del estudio de Carvalho y col. (12) que encuentran una frecuencia muy alta de sobremordidas, se puede apreciar, en líne- as generales, que en la dentición primaria las maloclusiones típicas son los apiñamientos, las mordidas cruzadas posteriores y las mordidas abiertas anteriores.

En este estudio parece confirmarse la influencia del tipo y duración de la crianza que se recibe siendo infante sobre el desarrollo del sistema estomatognático (14), fundamentalmente en el grupo de las niñas. Así cuanto más tiempo de lactancia natural tengan, menor es la prevalencia de las maloclusiones ( $p=0,0211)$. Incluso se da una relación inversamente proporcional entre la duración de la lactancia y la severidad de la maloclusión.

La hipótesis de que la malposición dificulta la higiene sólo se pudo confirmar en los niños $(p=0,0211)$. En la muestra se manifestó únicamente una tendencia a influir la maloclusión de los dientes en la higiene oral (Tabla 4).

En esta dentición, la caries no es tan determinante 


\section{TABLA 6.- FRECUENCIAS DE LAS MALOCLUSIONES SEGÚN DISTINTOS ESTUDIOS EN NIÑOS EUROPEOS}

\begin{tabular}{|l|c|c|c|c|}
\hline \multicolumn{1}{|c|}{ PREVALENCIAS } & $\begin{array}{c}\text { Estudio } \\
\text { Vigo, España } \\
\text { N=441 } \\
\text { Edad 1-7 años }\end{array}$ & $\begin{array}{c}\text { Carvalho y col. (12) } \\
\text { Leuven, Bélgica } \\
\text { N=750 } \\
\text { Edad 3-5 años }\end{array}$ & $\begin{array}{c}\text { Tschill y col. (13) } \\
\text { Strasbourg, Francia } \\
\text { N=789 } \\
\text { Edad 4-6 años }\end{array}$ & $\begin{array}{c}\text { Tapias y col. (8) } \\
\text { Móstoles, España } \\
\text { N=953 } \\
\text { Edad 6 años }\end{array}$ \\
\hline Apiñamiento anterior & $35,80 \%$ & $10,10 \%$ & $24,00 \%$ & $56,10 \%$ \\
Mordida cruzada posterior & $24,80 \%$ & $2,00 \%$ & $37,40 \%$ & $26,90 \%$ \\
Mordida abierta anterior & $20,80 \%$ & & $26,00 \%$ & $33,80 \%$ \\
Clase II de Angle & $20,80 \%$ & & & $10,00 \%$ \\
Clase III de Angle & $2,30 \%$ & & & \\
Resalte & $2,30 \%$ & $32,00 \%$ & \\
Sobremordida & $1,10 \%$ & $32,00 \%$ & \\
\hline
\end{tabular}

\begin{tabular}{|c|c|c|}
\hline \multicolumn{4}{|c|}{ TABLA 7.- DIFERENTES ESTUDIOS SOBRE LA } \\
PREVALENCIA DE LAS AGENESIAS DENTALES, \\
LOS DIENTES SUPERNUMERARIOS Y LAS FUSIONES \\
DENTALES
\end{tabular}

para la oclusión como en otras etapas (6). Algo que parece lógico, pues la pérdida de espacio que se puede producir sólo influye en la dentición definitiva. Tampoco en esta dentición parece ser determinante la presencia de un síndrome de obstrucción respiratoria, aunque se admite que la respiración nasal es un estímulo importante para el desarrollo óptimo del esqueleto maxilofacial.

La prevalencia de niños con agenesias dentales es de $0,90 \%$, con fusión dental es de 0,22. Estas anomalías tienen unas prevalencias similares en niños de países distintos. Una excepción ser observa en niños japoneses. Si estas anomalías tienen un componente hereditario importante, en el caso de los niños japoneses se puede pensar que es debido a un rasgo racial $y / o$ ambiental.

En esta serie se encontró la prevalencia más alta de dientes supernumerarios $(1,13 \%)$, curiosamente quienes presentan la prevalencia más pequeña en este tipo de anomalía son los niños japoneses (Tabla 7).

El diente que más se afectó fue el incisivo lateral. En el caso de agenesias dentales fue por igual en la mandíbula y en el maxilar. En el caso de dientes supernumerarios el $80 \%$ de los casos fueron en el maxilar, siendo uno de ellos un 


\section{TABLA 9.- PREVALENCIA DE LA CARIES DE BIBERÓN EN ESTUDIOS DE DIFERENTES PAÍSES}

\begin{tabular}{|l|c|c|c|}
\hline & MUESTRA & EDAD & PREVALENCIA \\
\hline Ostos Garrido y col. (28) España (1991) & 300 niños & $24-62$ meses & $12 \%$ \\
Sewint y col. (37) USA (1993) & 110 niños & $18-36$ meses & $20 \%$ \\
al-Dashti y col. (36) Kuwait (1995) & 227 niños & $18-48$ meses & $19 \%$ \\
Weerheijm y col. (18) Holanda (1998) & 96 niños & 28,8 meses media & $9,30 \%$ \\
Quartey y col. (18) USA (1999) & 135 niños & 57 meses media & $44 \%$ \\
\hline
\end{tabular}

incisivo central. En tres de cuatro casos con agenesia del incisivo lateral $(75 \%)$ se produjo agenesia en el homónimo permanente en los tres casos y además en dos faltaron más piezas permanentes. En un caso de agenesia del temporal no se produce ausencia de ninguno permanente. En tres de los cinco casos de dientes supernumerarios también se encontraron supernumerarios homónimos en la dentición permanente, en los dos casos restantes no se tuvo la oportunidad de realizar una ortopantomografía de control. Resultados parecidos se encuentran en estudios similares (12, 15, $17,18)$.

La prevalencia de niños con dientes natales en el estudio es de $0,22 \%$. Kates y col. (20) encuentran una prevalencia que oscila entre el $0,02 \%$ y el $0,13 \%$ según diferentes encuestas. Esta prevalencia aumentan en casos de labio leporino o hendidura del paladar (21). La mayoría de las veces son incisivos normales que se deben mantener excepto si presentan mucha movilidad.

\section{CONCLUSIONES}

1.- La prevalencia de las maloclusiones en esta dentición es alta, aunque se deben de unificar todavía más los criterios para conseguir datos fidedignos.

2.- La detección temprana de la maloclusión permite planificar el tratamiento a largo plazo según las necesidades de cada niño (12). En esta edad se deben de tratar las mordidas cruzadas laterales y las mordidas abiertas (13).

3.- Las otras anomalías dentales tienen una prevalencia muy baja, sin embargo, siempre deben de alertar sobre posibles alteraciones en la dentición permanente.

\section{BIBLIOGRAFÍA}

1. Gabinete de Estudios Sociológicos Bernard Krief. Estudio prospectivo Delphi. Libro Blanco. La salud bucodental en España. "Odonto-estomatología 2005”. Editado por Lacer. 1997.

2. Riobóo R. La evolución de la salud buco-dental en la población europea durante la última década: Variables implicadas. Av. Odontoestomatol 1998; 14: 119-133.

3. Jiménez Villa J, Grifell Martín E. Conceptos de estadística. En Atención Primaria. Concepto, organización, práctica clínica. De Martín Zurro A y Cano Pérez JF. Ed. Mosby/Doyma 3º ed. 1994. 236-55.

4. Pita Fernández S. Determinación del tamaño muestral. Cad Aten Primaria 1996; 3: 138-41.

5. Mayoral. Desarrollo de los dientes y de la oclusión. En Ortodoncia. Principios fundamentales y práctica. Ed. Labor S.A., $5^{a}$ ed. 1986, 59-86.

6. Iglesias Parada, IM. Influencia del sexo, tipo de lactancia, caries y síndrome de obstrucción respiratoria sobre la prevalencia de la maloclusión en la población infantil de Vigo con dentición mixta. Av Odontoestomatol 2000; 16: 251-263.

7. Cadena Galdós A, Hinojosa A. La maloclusión en la dentición primaria. Rev ADM 1990 May-Jun; 47 (3): 107-11. [Medline].

8. Tapias Ledesma MA, Gil de Miguel A, Domínguez Rojas V. Prevalencia de caries en una población infantil de Móstoles. Av Odontoestomatol 2000; 16: $241-250$. 
9. Lozano M, Ortiz A, de la Torre J, Cabello A, Gandul $R$. Encuesta en escolares de $1^{\circ}$ de EGB sobre maloclusiones/caries en la privincia de Córdoba, año 1992. Rev And Odontoestomatol 1993; (3): 188-193.

10. Legovic M, Mady L. Occlusal anomalies in the deciduous and mixed bites. Stomatologiia (mosk) 1998; 77 (5): 56-60. [Medline].

11. Moyers RE. Desarrollo de la dentadura y la oclusión. Cambios dimensionales en los arcos dentarios. En Manual de ortodoncia para el estudiante y odontólogo general. Editorial Mundi S.A.I.C. y F. $3^{\mathrm{a}}$ ed. 1985, 196-201.

12. Carvalho JC, Vinker F, Declerck D. Malocclusion, dental injuries and dental anomalies in the primary dentition of Belgian children. Int Paediatr Dent 1998 Jun; 8 (2): 137-41. [Medline].

13. Tschill P, Bacon W, Sonko A. Malocclusion in the deciduous dentition of Caucasian children. Eur J Orthod 1997 Aug; 19 (4): 361-7. [Medline].

14. Planas P. Génesis del sistema estomatognático bajo el concepto de la "Rehabilitación neuro-oclusal”. En Rehabilitación neuro-oclusal (RNO). Ed. Salvat S.A. 1987, 91-102.

15. Jarvinen S, Lehtinen L. Supernumerary and congenitally missing primary teeth in Finish children. An espidemiologis study. Acta Odontol Scand 1981; 39 (2): 83-6. [Medline].

16. Skrinjaric I, Barac-Furtinovic V. Anomalies of deciduous teeth and findings in permanent dentition.
Acta Stomatol Croat 1991; 25 (3): 151-6. [Medline].

17. Yonezu T, Hayashi Y, Sasaki J, Machidad Y. Prevalence of congenital dental anomalies of the deciduous dentition in Japanese children. Bull Tokyo Dent Coll 1997 Feb; 38 (1): 27-32. [Medline].

18. Whinttington BR, Durward CS. Survey of anomalies in primary teeth and their correlation with the permanent dentition. N Z Dent J 1996 Mar; 92 (407): 4-8. [Medline].

19. Nik-Hussein NH, Abdul Majid Z. Dental anomalies in the primary dentition: distribution and correlation with the permanent dentition. J Clin Pediatr Dent 1996 Fall; 21 (1): 15-9. [Medline].

20. Kates GA, Needleman HL, Holmes LB. Natal and neonatal teeth; a clinical study. J Am Dent Assoc 1984 Sep; 109 (3): 441-3. [Medline].

21. de Almeida CM, Gomide MR. Prevalence of natal/neonatal teeth in cleft lip and palate infants. Cleft Palate Craniofac J 1996 Jul; 33 (4): 297-9. [Medline].

\section{CORRESPONDENCIA}

Isidro Manuel Iglesias Parada

C/ León, 2, $1^{\circ} \mathrm{B}$

Telf. 986412148

36205 Vigo 
